\title{
High Altitude-Induced Testicular Infarct: a Rare Occupational Hazard
}

\author{
Padma Deskit $^{1}{ }^{\mathbb{D}} \cdot$ Maneesha Badwal $^{1}$ - P. T. Wangchuk ${ }^{1}$
}

Received: 20 December 2021 / Accepted: 28 January 2022 / Published online: 2 February 2022

(c) Association of Surgeons of India 2022

\begin{abstract}
Visit to high altitude with its hypoxic hypobaric environment poses various physiological and biochemical challenges to a human body. If the body is not able to cope up to this changed environment, it leads to various maladies of high altitude like high altitude cerebral oedema, high altitude pulmonary oedema and spontaneous vascular thrombosis. There are reports of high altitude-induced deep venous thrombosis, pulmonary embolism, mesenteric ischemia etc. We report a unique case of high altitude-induced testicular infarct in a previously healthy individual after a brief stay at the high-altitude region Ladakh.
\end{abstract}

Keywords High altitude $\cdot$ Vascular thrombosis · Testicular infarct · Orchitis

\section{Introduction}

For several years, Ladakh is an inhabited hostile terrain. This high-altitude region has a stressful environment consisting of hypobaric hypoxia, low temperature, strong dry and chilly wind and strong ultraviolet radiation which poses challenge to the visitors from low altitude. These factors result in various physiological and biochemical stress to the human body. And in an effort to maintain the homeostasis in the body, various adaptive changes occur. If adaptive responses are inadequate, the visitors are subjected to various high altitude-related maladies [1]. One such condition is arterial and venous thrombosis. Thrombogenic effect of high altitude is not well known amongst low Landers. With Ladakh becoming a popular tourist destination, our paper is an effort to throw light about this condition.

\section{Case Report (Figs. 1 and 2)}

A 26-year-old male originally hailing from Jharkhand landed at Leh (3500 m AMSL) in mid-April by air. He was hired to work as labourer around the Indo China border, in the Changthang region (4000 m AMSL). After $72 \mathrm{~h}$ at Leh for acclimatisation, he along with his friends was relocated for road building around the ALC. Whilst acclimatising, he

Padma Deskit

drpadmadeskit@gmail.com

1 SNM Hospital, Leh, Ladakh 194101, India showed no symptoms suggestive of high altitude cerebral oedema and high altitude pulmonary oedema. Barring few problems like sun burn, constipation and breathlessness during exertion, no other acute problems were encountered for initial 5 and half months. After 7 and half months of stay, he developed dull aching pain in his left scrotum, which was insidious in onset, continuous and partially relieved on taking rest and analgesics. He had no history of fever, burning micturition, trauma or sexual contact or associated abdominal pain and nausea. After 1 week of persistent pain, he went with the above symptoms to a primary health centre located about $30 \mathrm{~km}$ away, where doctor diagnosed him as a case of orchitis and put him on oral quinolones and anti-inflammatory. Seven days after, he was referred to a district hospital at Leh for persistence of symptoms. His urine examination done at the primary health centre showed 2-3 pus cells.

On arrival at our hospital, he complained of persistent, continuous pain. His vitals were stable. The patient's left testis was firm to hard and tender on palpation. Since his tenderness increased on elevation, we suspected neglected torsion testis. Ultrasound scrotum shows left testicular infarct and no Doppler flow in the left testicular artery. His blood investigation showed a haemoglobin of $19 \mathrm{gm} \%$, haematocrit of $62.4 \%$ and mean platelet volume of $13.5 \mathrm{fl}$ and D dimer was moderately elevated. Prothrombin time was low (10.7 s). And RT-PCR for SARS-CoV-2 was negative. Repeat urine examination done showed $3-4$ pus cell and no other significant finding.

The patient was posted for orchidectomy under spinal anaesthesia, no torsion was noted, and the testis and 


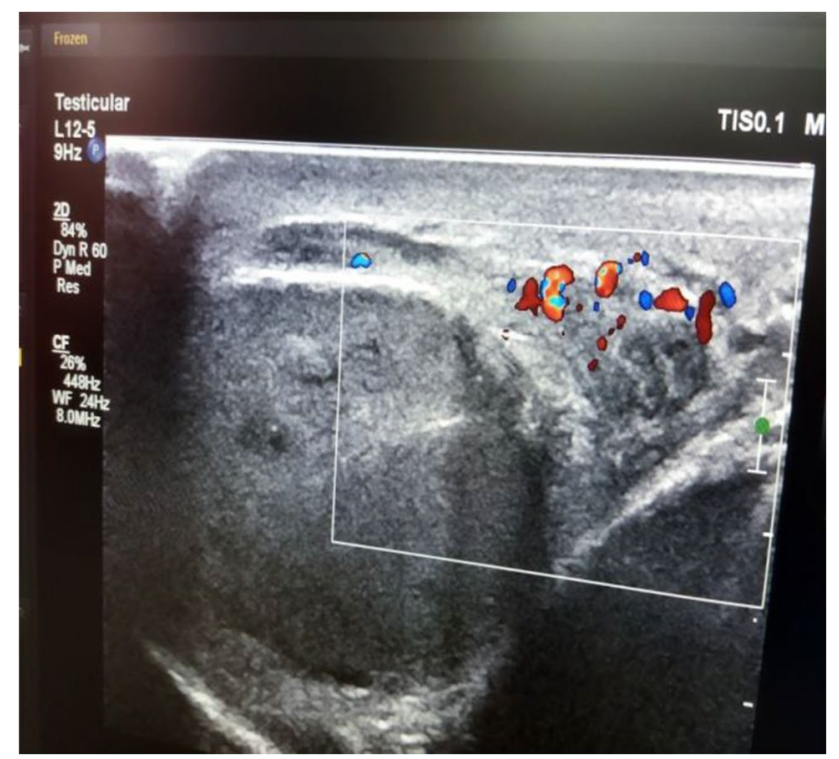

Fig. 1 Ultrasound image showing left testis with ill-defined hypo echoic area and left hydrocele with minimal vascularity only around mediastinum testis

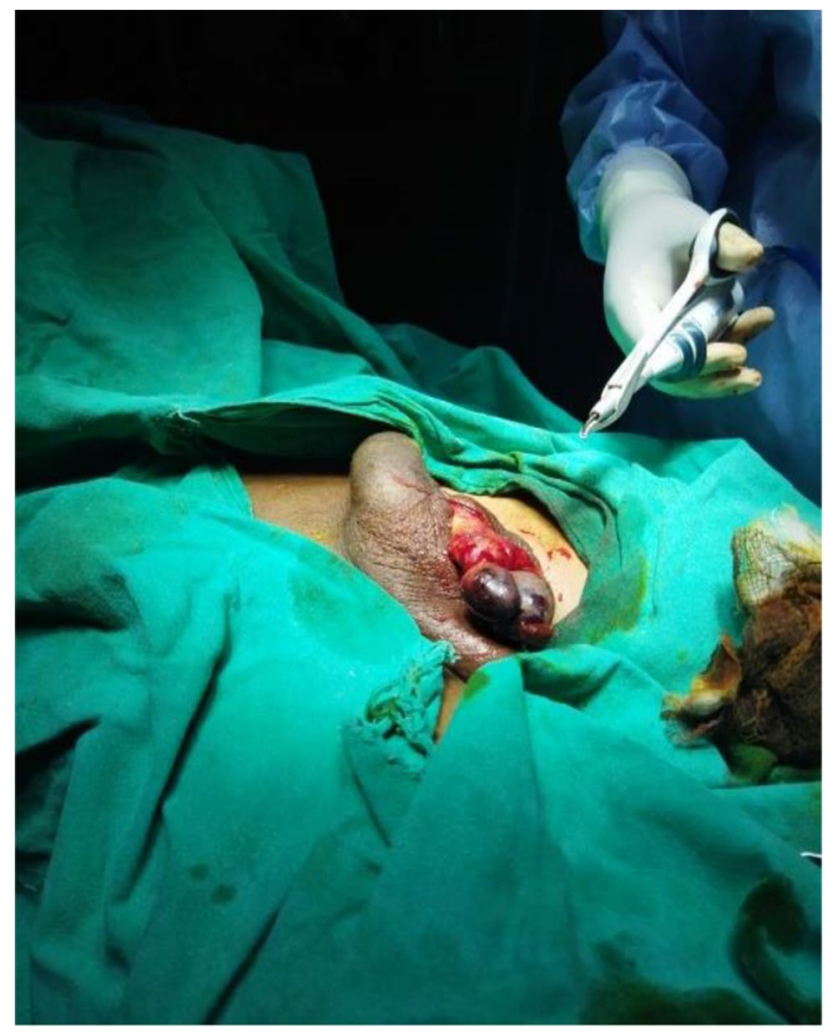

Fig. 2 Pre-operative image of gangrenous and enlarged testis and epididymis

epididymis were gangrenous. Orchidectomy was done and in post-operative period the patient was put on LMW heparin.
The post-operative period was uneventful and the patient was put on aspirin and oral anticoagulant in view of polycythemia and deranged prothrombin time assay, and was advised to refrain from going back to Changthang and other higher altitude places. Post-operatively, preliminary Doppler ultrasound of abdominal vessels was normal. Patient was doing well at 1 week and month follow-up.

\section{Discussion}

Thrombogenic nature of high altitude is a known entity but still needs a lot of systematic study and research [2]. Both arterial and venous thrombosis occur at high altitude. Thirty times increased risk of spontaneous vascular thrombosis amongst soldier stationed at high altitude has been reported [3].Various theories have been suggested for the possible cause. As report and seen in our case of erythrocytosis, elevated platelet level and increased platelet activation and raised fibrinogen level along with hypoxia and dehydration create a thrombotic milieu [4]. Chohan et al. reported that hypercoagulability on arrival at high altitude is evidenced by increased platelet count, factor X and XII level [5]. Though both arterial and venous systems are affected, there are more studies on venous thrombosis [6,7]. Arterial thrombosis has been reported in studies in the form of stroke and pulmonary infarct [8]. There is a report of thrombosis in the aorta with limb ischemia, and multifocal arterial thrombosis in pulmonary artery and superior mesenteric artery [9]. Using various search engines, we could only find cases reporting about thrombosis in pampiniform plexuses in patients with underlying thrombophilia due to various causes, but no case report of isolated thrombosis in testicular artery due to high altitude has been reported. On follow-up after 1 month, the patient was doing well.

An interesting study on mechanism of cellular and molecular changes which occur in high altitude-exposed testis suggested that if compensatory mechanism like in neoangiogenesis increased erythropoietic production under the influence of hypoxia-induced factor, and if hypobaric hypoxia continues, it results in oxidative heat stress with resultant production of reactive oxygen and nitrogen species, leading to enzymatic activation and apoptosis and testicular injury [10].

Studies on the effect of high altitude on humans are difficult and negligible making the management of such case difficult. A positive study in this direction suggested that such testicular damage at high altitude was mediated by serotonin, whose level was seen to increase as early as after 4 days of exposure to high altitude, and also experimentally testicular damage could be prevented in rats by previous administration of serotonin antagonist cyproheptadine [11]. 


\section{Conclusion}

Since there is no single diagnostic test for these vascular events, it requires high suspicion and greater awareness on the part of clinician to detect the case at earliest. Slow ascend, avoiding vigorous exercises, maintaining good hydration, avoiding alcohol and picking up any predisposing genetic factor before induction can save life, limbs and gonads as in our case.

Acknowledgements The authors extend gratefulness to the patient who gave us the consent.

\section{Declarations}

Conflict of Interest The authors declare no competing interests.

\section{References}

1. Wilson MH, Newman S, Imray CH (2009) The cerebral effects of ascent to high altitudes. Lancet NEUROL 8:175-191

2. Gambir RPS, Anand V, Khatana SS, Bedi VS (2014) A brief review of high altitude thrombosis. Indian J Endovasc Surg 1:20

3. Anand AC, Jha SK, Saha A, Sharma V, Adya CM (2001) Thrombosis as a complication of extended stay at high altitude. Natl Med J Indis 14(4):197-201
4. Kotwal J, Apte CV, Kotwal A, Mukherjee B, Jayaram J (2007) High altitude: a hypercoagulable state: result of a prospective cohort study. Thromb Res 120(3):391-397. https://doi.org/10. 1016/j.thromres.2006.09.013

5. Chohan IS (1984) Blood coagulation changes in high altitude. Def Sci J 34:360-362

6. Kumar S (2006) High altitude induced deep venous thrombosis: a study of 28 cases. Indian J Surg T 68:84-88

7. Torgovicky R, Azaria B, Grossman A, Eliyahu U, Goldstein L (2005) Sinus vein thrombosis following exposure to simulated high altitude. Aviat Space Environ Med 76:144-146

8. Fagenholz PJ, Gutman JA, Murray AF, Noble VE, Wu A, Zeimer G (2007) Arterial thrombosis at high altitude resulting in loss of limb. High Altitude Med Biol 8(4):340-347

9. McKay D, Marron C, Brown R (2021) Testicular infarction secondary to protein S deficiency: a case report. BMC Urol 21:174

10. Reyes JG, Farias JG, Henriquez-Olavarrieta S, Madrid E, Parraga M, Zepeda AB, Moreno RD (2012) The hypoxic testicle: physiology and pathophysiology. Oxid Med Cell Longev 2012:929285. https://doi.org/10.1155/2012/929285

11. Gonzales GF, Rodriguez L, Valera J, Sandoval E, Garcia-Hjarles M (1990) Prevention of high altitude induced testicular disturbances by previous treatment with cyproheptadine in male rats. Ach Androl 24(2):201-2015. https://doi.org/10.3109/0148501900 8986880

Publisher's Note Springer Nature remains neutral with regard to jurisdictional claims in published maps and institutional affiliations. 